\title{
NONLINEAR DEFLECTION OF A THIN ELASTIC SHEET ON A LIQUID FOUNDATION*
}

\author{
BY \\ CHANG-YI WANG \\ Michigan State University
}

\begin{abstract}
A thin infinite elastic sheet lies on the surface of a liquid. The nonlinear deflection due to a concentrated load is studied by both analytical and numerical methods.
\end{abstract}

Introduction. Elastic beams or sheets on a foundation are important in many practical engineering models, including highways, railroads, and floating bridges [1]. The usual analyses assume infinitesimal deflections, resulting in a more solvable linear problem. The large deflection problem has never been investigated, although it does occur in the case of pliable foundations such as sand, mud or water. For such foundations the resistance to deformation is due to hydrostatic pressure.

Figure 1 shows an infinite elastic sheet lying on a liquid foundation. We shall consider two dimensional deformations due to a vertical point load.

Foundation. We assume the thickness of the sheet is small compared to a characteristic length, say maximum deflection $b^{\prime}$. Then the sheet can be considered as an elastica, i.e. the local curvature is proportional to the local applied moment $m$ :

$$
m=E I \frac{d \theta}{d s^{\prime}} .
$$

Here $E I$ is the flexural rigidity, $\theta$ is the local angle of inclination and $s^{\prime}$ is the arc length from the point force of magnitude $2 F^{\prime}$. Let Cartesian axes $\left(x^{\prime}, y^{\prime}\right)$ be located as shown in Fig. 1. The unperturbed liquid surface originally lies at $y^{\prime}=0$. Then

$$
\begin{aligned}
\frac{d x^{\prime}}{d s^{\prime}} & =\cos \theta, \quad \frac{d y^{\prime}}{d s^{\prime}}=-\sin \theta, \\
x^{\prime}(0) & =0, \quad y^{\prime}(0)=b^{\prime}, \quad y^{\prime}(\infty)=0 .
\end{aligned}
$$

Figure 1 also shows an elemental length $d s^{\prime}$. A balance of local moments gives the leading terms

$$
m+d m=m-\left[\int_{s^{\prime}}^{\infty} p \cos \theta d s^{\prime}\right] d s^{\prime} \cos \theta+\left[H^{\prime}-\int_{s^{\prime}}^{\infty} p \sin \theta d s^{\prime}\right] d s^{\prime} \sin \theta
$$




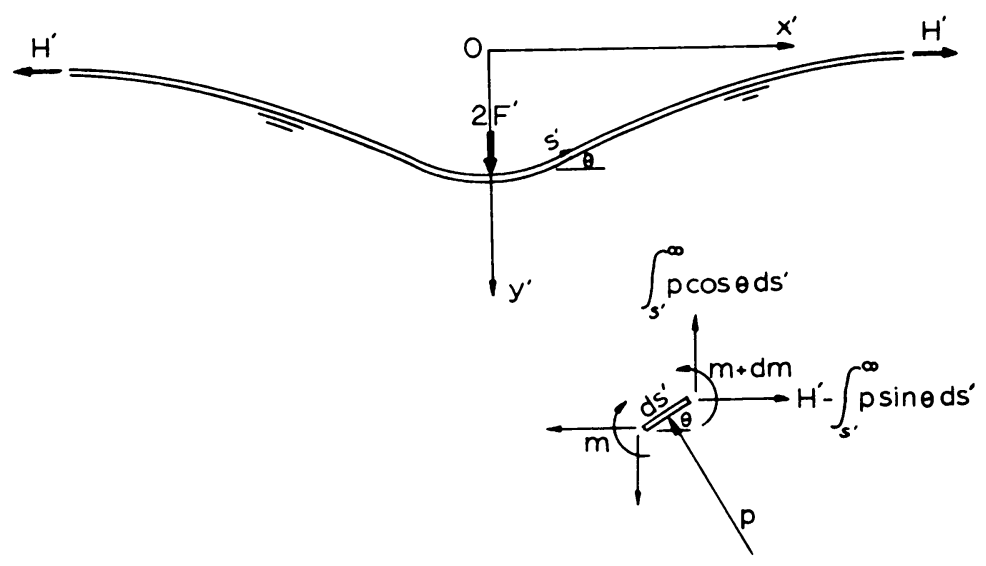

FIG. 1. The coordinate system and the forces on an elemental length.

where $H^{\prime}$ is the horizontal force at infinity and $p$ is the local pressure difference given by

$$
p=\rho g y^{\prime} \equiv k y^{\prime} .
$$

Here $\rho$ is the liquid density and $g$ is the gravitational acceleration. Using Eqs. (2)-(5) we find

$$
E I \frac{d^{2} \theta}{d s^{\prime 2}}=\left(k \int_{0}^{s^{\prime}} y^{\prime} \cos \theta d s^{\prime}-F^{\prime}\right) \cos \theta+\left[H^{\prime}-\frac{k}{2}\left(y^{\prime}\right)^{2}\right] \sin \theta .
$$

If neither $E I$ nor $k$ is zero, we normalize all lengths by $(E I / k)^{1 / 4}$, all forces by $\sqrt{E I k}$ and drop primes. The governing equations become

$$
\begin{aligned}
& \frac{d^{2} \theta}{d s^{2}}=(u-F) \cos \theta+\left(H-\frac{1}{2} y^{2}\right) \sin \theta, \\
& \frac{d u}{d s}=y \cos \theta, \quad \frac{d x}{d s}=\cos \theta, \quad \frac{d y}{d s}=-\sin \theta .
\end{aligned}
$$

The boundary conditions are

$$
\begin{aligned}
\theta(0)=0, & u(0)=0, \quad x(0)=0, \quad y(0)=b, \\
\theta(\infty)=0, & u(\infty)=F, \quad y(\infty)=0 .
\end{aligned}
$$

This set of equations is highly nonlinear and exact solution does not exist. Given $F, H$ the unknowns are $\theta, d \theta / d s, u, x, y, b$.

The perturbation solution for small $F$. Small $F$ indicates the applied load is small compared to $\sqrt{E I k}$. A study of eqs. (7)-(10) shows $u, y, \theta$ are small too. We expand the unknowns in a power series in $F$ as follows

$$
\begin{aligned}
& \theta=F \theta_{0}+F^{3} \theta_{1}+\ldots, \\
& u=F u_{0}+F^{3} u_{1}+\ldots, \\
& y=F y_{0}+F^{3} y_{1}+\ldots, \\
& x=x_{0}+F^{2} x_{1}+\ldots
\end{aligned}
$$


Equations (7)-(10) yields zeroth order equations.

$$
\begin{aligned}
\frac{d^{2} \theta_{0}}{d s^{2}} & =u_{0}-1+H \theta_{0}, \\
\frac{d u_{0}}{d s} & =y_{0}, \quad \frac{d x_{0}}{d s}=1, \quad \frac{d y_{0}}{d s}=-\theta_{0}, \\
\theta_{0}(0) & =0, \quad u_{0}(0)=0, \quad x_{0}(0)=0, \\
\theta_{0}(\infty) & =0, \quad u_{0}(\infty)=1, \quad y_{0}(\infty)=0 .
\end{aligned}
$$

Equation (12) is differentiated once to yield

$$
\frac{d^{4} y_{0}}{d s^{4}}-H \frac{d^{2} y_{0}}{d s^{2}}+y_{0}=0
$$

If $H=0$ Eq. (15) becomes the classical equation for a beam on an elastic foundation [2].

Without going into details, the solution for $H>-2, H \neq 2$ is

$$
\begin{aligned}
& y_{0}=\frac{1}{\sqrt{H^{2}-4}}\left(\beta e^{\alpha s}-\alpha e^{\beta s}\right), \\
& u_{0}=\frac{1}{\sqrt{H^{2}-4}}\left(\frac{\beta}{\alpha} e^{\alpha s}-\frac{\alpha}{\beta} e^{\beta s}\right)+1, \\
& \theta_{0}=-\frac{1}{\sqrt{H^{2}-4}}\left(e^{\alpha s}-e^{\beta s}\right), \\
& x_{0}=s,
\end{aligned}
$$

where

$$
\left.\begin{array}{l}
\alpha \\
\beta
\end{array}\right\}=-\left(\frac{1}{2} H \pm \frac{1}{2} \sqrt{H^{2}-4}\right)^{1 / 2} \text {. }
$$

If $2>H>-2$, oscillatory decay occurs. The maximum height, occuring at $s=$ $2(2-H)^{-1 / 2} \pi$, is

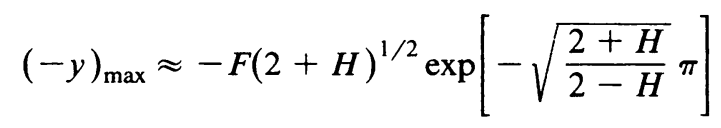

If $H \geqslant 2$ the solutions decay monotonically. For $H=2$, the solution is

$$
\begin{aligned}
& y_{0}=\frac{1}{2}(1+s) e^{-s}, \\
& u_{0}=1-\frac{1}{2} s e^{-s}-e^{-s}, \\
& \theta_{0}=\frac{1}{2} s e^{-s}, \\
& x_{0}=s .
\end{aligned}
$$

No decay solution exists for $H \leqslant-2$. The force-displacement relation for $H>-2$ is

$$
F=b \sqrt{H+2} \text {. }
$$


The first order perturbation equations are

$$
\begin{gathered}
\frac{d^{2} \theta_{1}}{d s^{2}}-u_{1}-H \theta_{1}=-\frac{1}{2}\left(u_{0}-1\right) \theta_{0}^{2}-H \frac{\theta_{0}^{3}}{6}-\frac{1}{2} \theta_{0} y_{0}^{2}, \\
\frac{d u_{1}}{d s}-y_{1}=-\frac{1}{2} \theta_{0}^{2} y_{0}, \\
\frac{d y_{1}}{d s}+\theta_{1}=\frac{\theta_{0}^{3}}{6}, \\
\frac{d x_{1}}{d s}=-\frac{1}{2} \theta_{0}^{2}, \\
\theta_{1}(0)=u_{1}(0)=x_{1}(0)=\theta_{1}(\infty)=u_{1}(\infty)==_{1}(\infty)=0 .
\end{gathered}
$$

It can be shown that the first order solutions are similar decaying exponentials. Higher order solutions can also be carried out. Since we are basically concerned with large deflections in this paper, the higher order linear corrections will not be persued.

Analysis of the nonlinear equations. Although Eqs. (7)-(10) cannot be solved explicitly, some important information can be obtained from its first integrals. We multiply Eq. (7) by $d \theta / d s$ and integrate with respect to $s$

$$
\begin{aligned}
\frac{1}{2}\left(\frac{d \theta}{d s}\right)^{2} & =\int(u-F) \cos \theta \frac{d \theta}{d s} d s+\int\left(H-\frac{1}{2} y^{2}\right) \sin \theta \frac{d \theta}{d s} d s \\
& =(u-F) \sin \theta-\int y \sin \theta \cos \theta d s-\left(H-\frac{1}{2} y^{2}\right) \cos \theta-\int \cos \theta y \frac{d y}{d s} d s \\
& =(u-F) \sin \theta-\left(H-\frac{1}{2} y^{2}\right) \cos \theta+C
\end{aligned}
$$

Using the boundary conditions at infinity we find the integration constant $C$ is $H$. At $s=0$ Eq. (23) yields

$$
d \theta / d s(0)=b
$$

If we multiply Eq. (7) by $y$ and integrate, the result is

$$
y \frac{d \theta}{d s}-\cos \theta=\frac{1}{2} u^{2}-F u-H \frac{y^{2}}{2}+\frac{y^{4}}{8}+C^{\prime},
$$

The far field boundary conditions give $C^{\prime}=F^{2} / 2-1$. Using Eqs. (9), (24), (25) we find

$$
b^{4}-4(H+2) b^{2}+4 F^{2}=0
$$

or

$$
b=\left[2(H+2) \pm 2 \sqrt{(H+2)^{2}-F^{2}}\right]^{1 / 2} .
$$

Due to symmetry, we consider positive $F$ and positive $b$ without loss of generality. A similarity form of Eq. (27) is

$$
\left(\frac{F}{H+2}\right)=\left(\frac{b}{\sqrt{H+2}}\right)\left[1-\frac{1}{4}\left(\frac{b}{\sqrt{H+2}}\right)^{2}\right]^{1 / 2} \text {. }
$$


This force-displacement relation is plotted in Fig. 2. The linear theory, represented by the dashed line, is valid for small $F$ only. Our nonlinear results show, as $b$ is increased, $F$ increases to a maximum at $b=\sqrt{2 H+4}$ where $F=H+2$. Then $F$ decreases as $b$ is further increased. Since negative slope implies instability, we conclude that only the rising part (for which $b<\sqrt{2 H+4}$ ) represent stable equilibrium states which can be observed in reality. Thus for given $H, F$ can be increased until $H+2$. The whole system would "sink" completely into the liquid if the vertical force $F$ is greater than $H+2$. In terms of dimensional variables the maximum load that can be sustained by an infinite elastic sheet on a liquid is

$$
\left(2 F^{\prime}\right)_{\max }=2 H^{\prime}+4 \sqrt{E I \rho g} .
$$

Note also that $H$ can be negative i.e. the horizontal force can be compressive. Suppose $F=0$ and the sheet is under compressive forces at infinity. Our solutions show

$$
H^{\prime}=-2 \sqrt{E I \rho g}
$$

is the critical buckling force for this infinite sheet.

The membrane. In this case the flexural rigidity $E I$ is zero. We renormalize all lengths by $\left(H^{\prime} / k\right)^{1 / 2}$ and assume neither $H^{\prime}$ nor $k$ are zero. Equation (6) reduces to

$$
\begin{gathered}
(\tilde{u}-\alpha) \cos \theta+\left(1-\frac{1}{2} \tilde{y}^{2}\right) \sin \theta=0, \\
\frac{d \tilde{u}}{d \tilde{s}}=\tilde{y} \cos \theta, \quad \frac{d \tilde{x}}{d \tilde{s}}=\cos \theta, \quad \frac{d \tilde{y}}{d \tilde{s}}=-\sin \theta,
\end{gathered}
$$

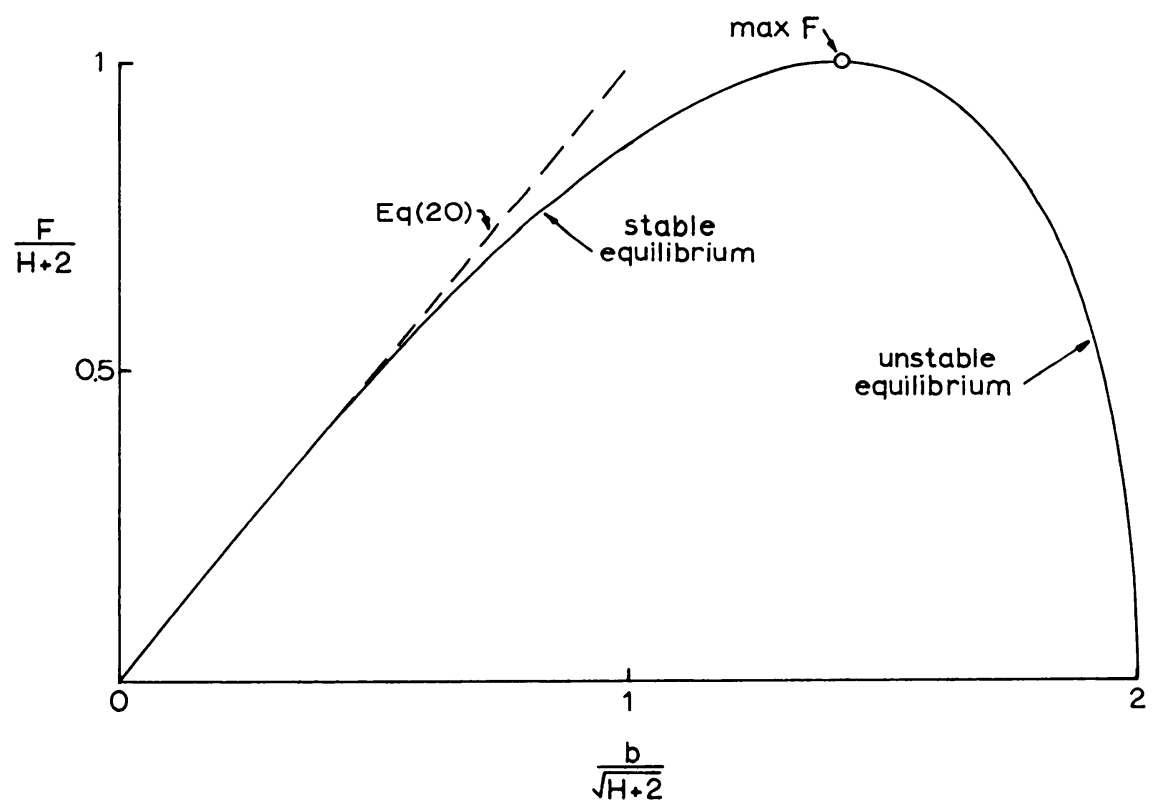

FIG. 2. The exact force-displacement relation. 
where the tildes represent the renormalized variables and $\alpha \equiv F^{\prime} / H^{\prime}$. The appropriate boundary conditions are

$$
\begin{gathered}
\tilde{u}(0)=\tilde{x}(0)=0, \quad \tilde{y}(0)=\tilde{b}, \\
\tilde{u}(\infty)=\alpha, \quad \theta(\infty)=\tilde{y}(\infty)=0 .
\end{gathered}
$$

Equations (31)-(34) are still nonlinear. However, exact analytic solution is possible. We differentiate Eq. (31) once to give

$$
(\tilde{u}-\alpha) d \theta / d \tilde{s}=\tilde{y} \sin \theta .
$$

Utilizing

$$
\frac{d \theta}{d \tilde{s}}=\frac{d \tilde{u}}{d \tilde{s}} \frac{d \theta}{d \tilde{s}}=\tilde{y} \cos \theta \frac{d \theta}{d \tilde{u}} .
$$

Equation (35) is integrated to be

$$
\tilde{u}=\alpha+C^{\prime \prime} \sin \theta
$$

where $C^{\prime \prime}$ is a constant of integration. Then from Eq. (31)

$$
\frac{1}{2} \tilde{y}^{2}=1+C^{\prime \prime} \cos \theta
$$

The boundary conditions at infinity yield $C^{\prime \prime}=-1$. Using Eqs. (35)-(37)

$$
\frac{d \theta}{d \tilde{s}}=-\tilde{y}=-\sqrt{2-2 \cos \theta}=-2 \sin \frac{\theta}{2} .
$$

A further integration results in

$$
\tilde{s}=-\ln \frac{\tan (\theta / 4)}{\tan \left(\theta_{0} / 4\right)}
$$

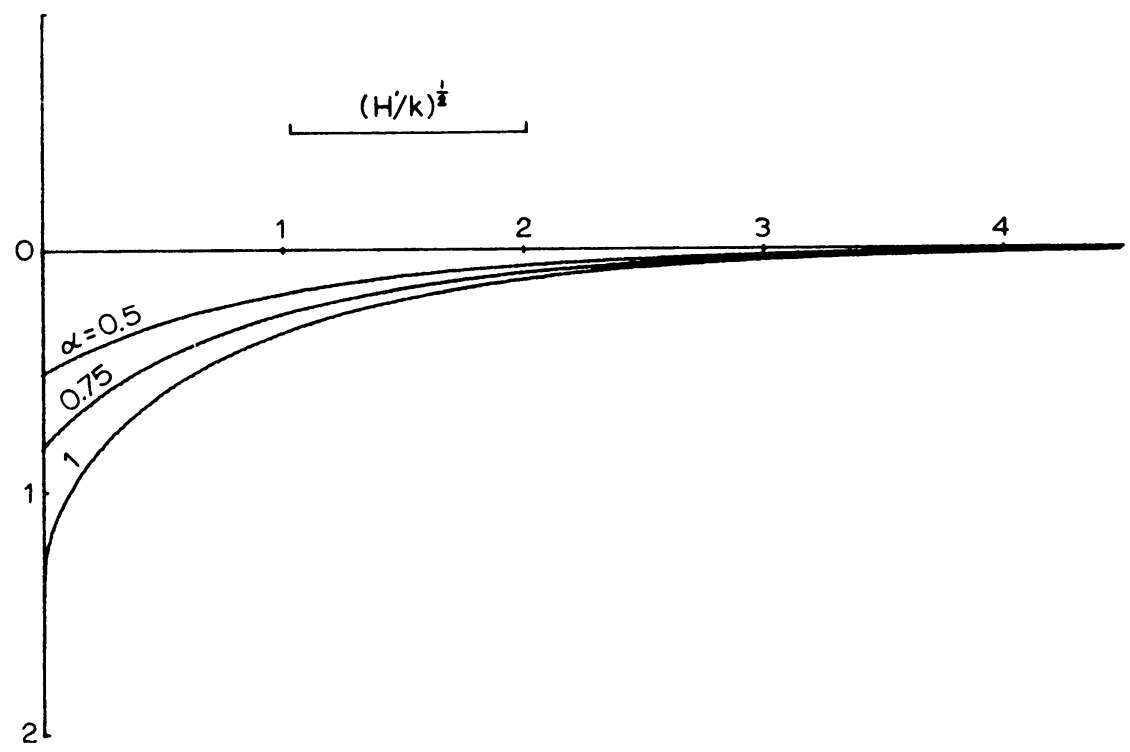

FIG. 3. Surface configurations for concentrated force on a membrane. 
where $\theta_{0}$ is the value of $\theta$ at $\tilde{s}=0$. From Eq. (37)

$$
\theta_{0}=\sin ^{-1} \alpha \text {. }
$$

Equations (32), (39), (40) can be integrated to give the configuration of the sheets in parametric form.

$$
\begin{gathered}
\tilde{x}=-\ln \frac{\tan (\theta / 4)}{\tan \left(\theta_{0} / 4\right)}-2 \cos \frac{\theta}{2}+2 \cos \frac{\theta_{0}}{2}, \\
\tilde{y}=2 \sin \frac{\theta}{2} .
\end{gathered}
$$

Figure 3 shows some surface configurations for various $\alpha$. The force-displacement relation is

$$
\tilde{b}=2 \sin \frac{\theta_{0}}{2}=\left(2-2 \sqrt{1-\alpha^{2}}\right)^{1 / 2} .
$$

This relation shows $\alpha$ can be at most 1 (or $F^{\prime} \leqslant H^{\prime}$ ). It is also consistent with Eq. (27) in the limit of $E I \rightarrow 0$. The amount of shortening at infinity is

$$
\tilde{s}-\left.\tilde{x}\right|_{\tilde{s} \rightarrow \infty}=2-2 \cos \frac{\theta_{0}}{2}=2-\left(2+2 \sqrt{1-\alpha^{2}}\right)^{1 / 2} .
$$

Numerical integration. For general $E I$, the shape of the surface must be obtained by numerical integration of Eqs. (3)-(10). We turn this two-point boundary value problem into an initial value problem by supplanting Eq. (10) by Eqs. (24) and (27). The bottom sign of Eq. (27) is taken due to stability reasons discussed previously. The integration uses a fourth order Runge-Kutta algorithm where a step size of 0.05 is found to be sufficient.

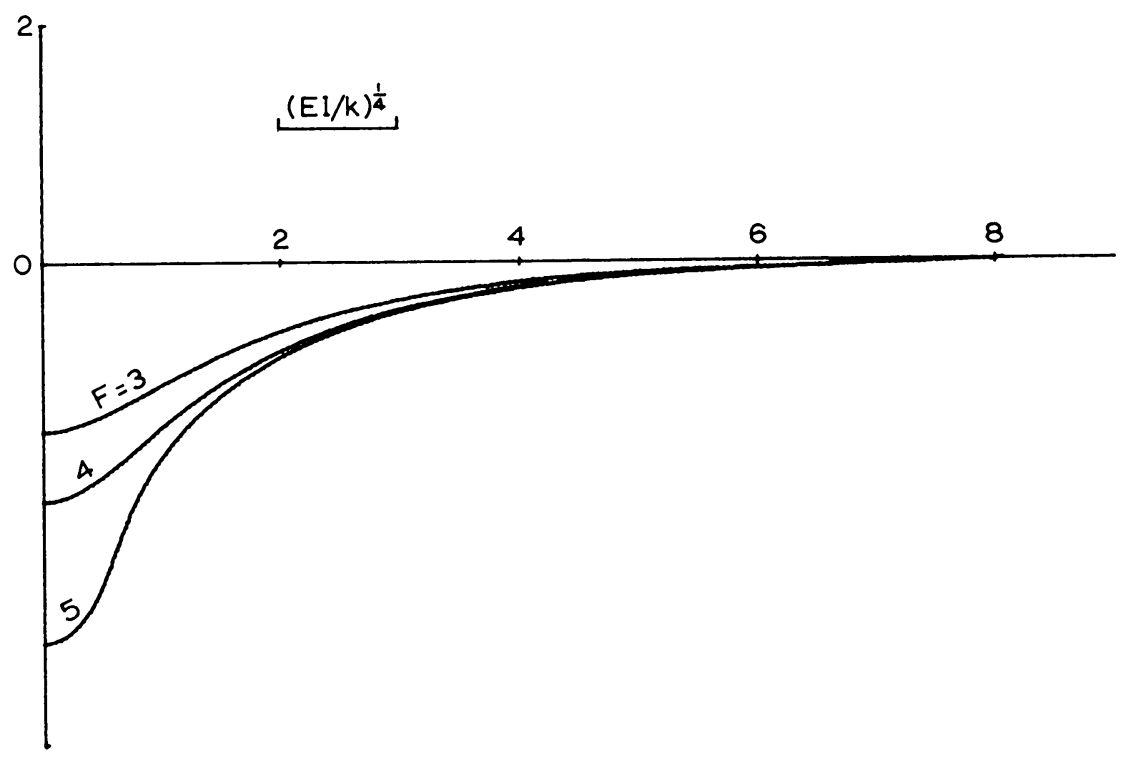

Fig. 4. Surface configuration for $H=3$. Maximum load is $F=5$. 


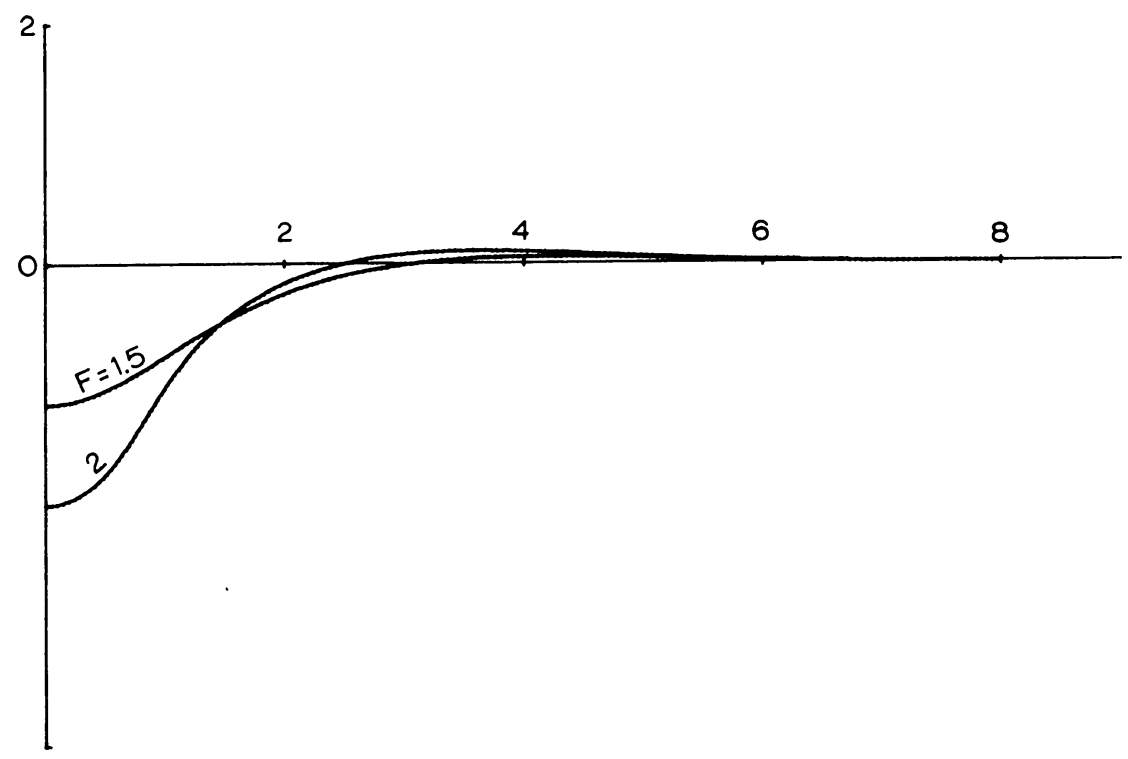

FIG. 5. Surface configuration for $H=0$. Maximum load is $F=2$.

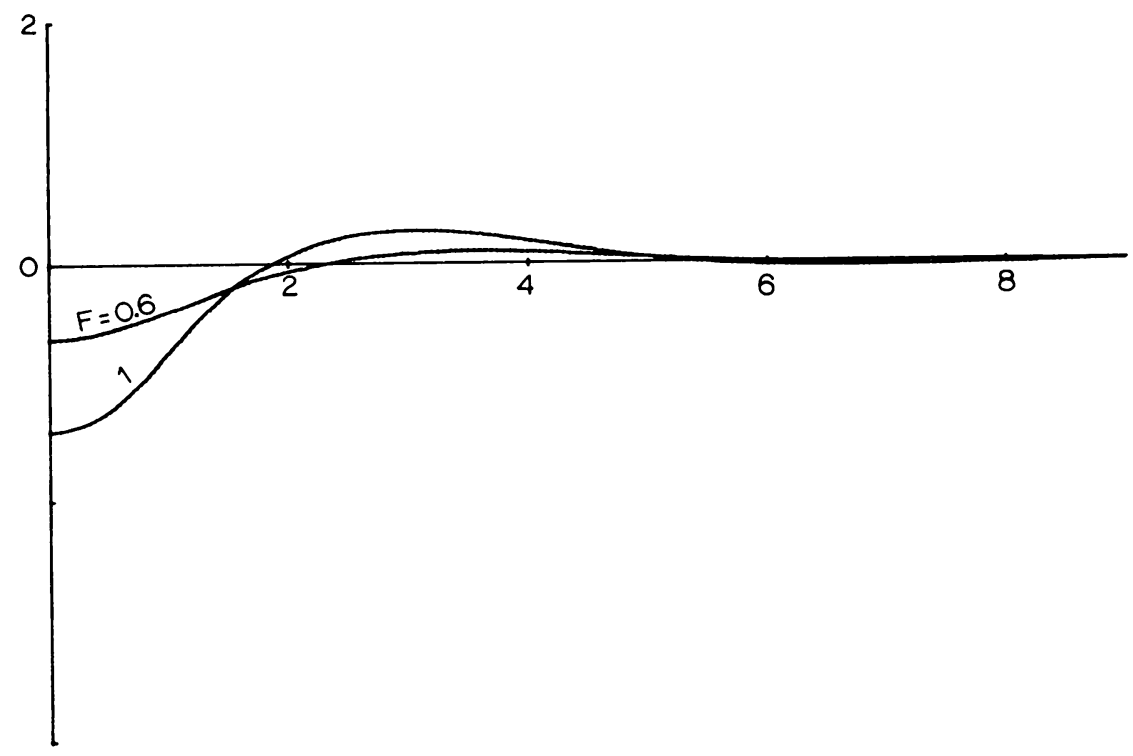

Fig. 6. Surface configuration for $H=-1$. Maximum load is $F=1$.

Figure 4 shows half of the surface for $H=3$ and various $F$. Note for large $F$ and $H$ ( $E I$ small) the shape approaches to that of Fig. 3, except for a region near $s=0$. The shape is consistent with our linear analysis which predicted monotonic approach to $y=0$ as $s$ is increased. The maximum stable load in this case is $F=5$ above which the sheet, applied forces and all, will sink continuously into the fluid. Figure 5 shows the case $H=0$ or no 


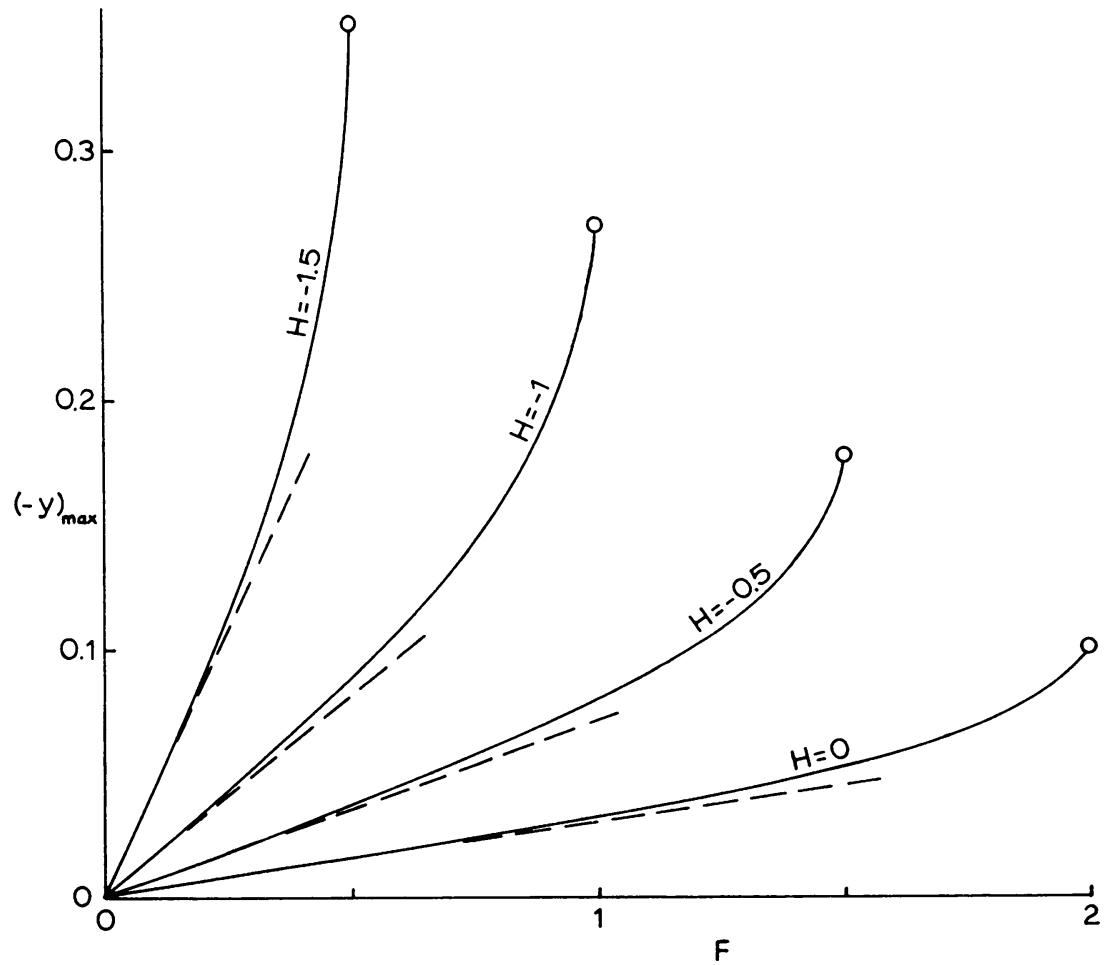

FIG. 7. Maximum rise as a function of load $F$. Dashed lines are the linear approximation Eq. (18). Circle indicates maximum stable load.

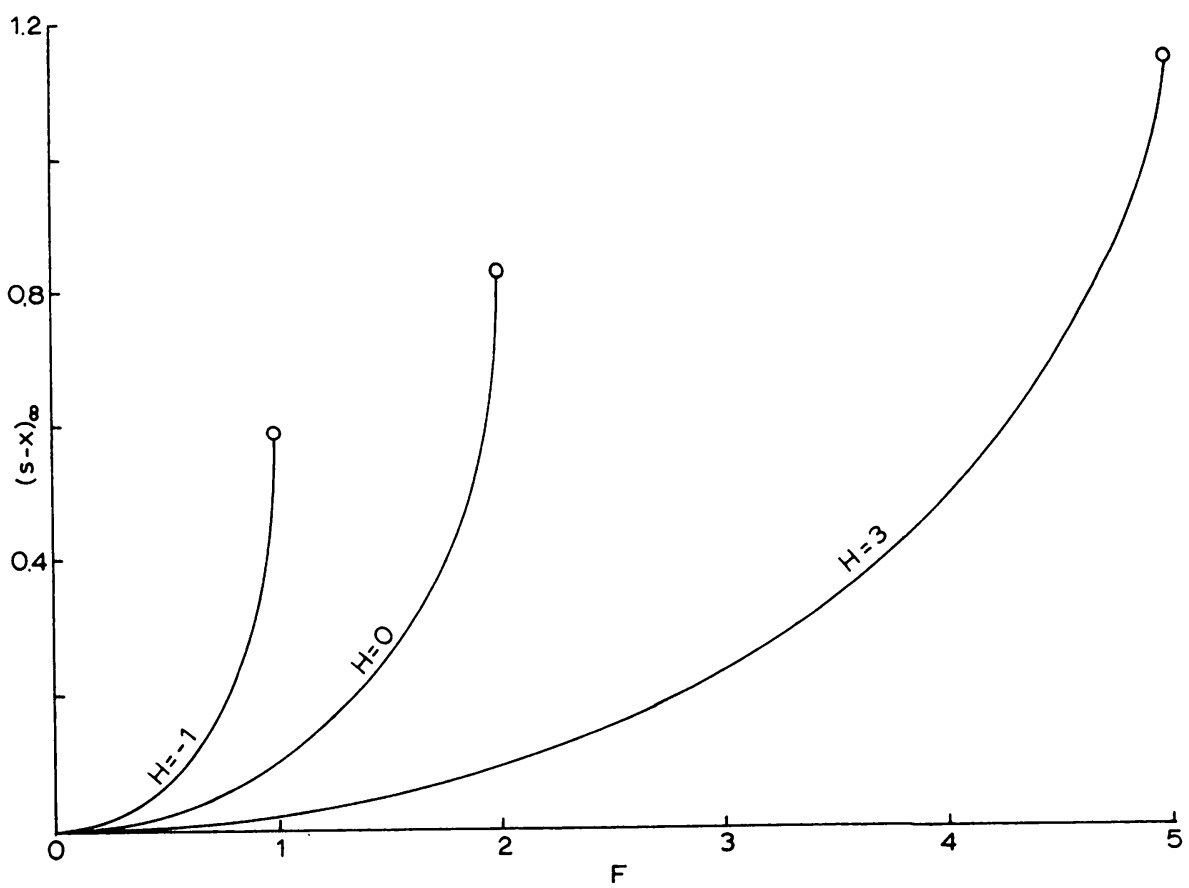

FIG. 8. Shortening at infinity as a function of load $F$. 
horizontal force. The rigidity $E I$ now plays an important role to maintain equilibrium. Figure 6 shows the case for a compressive force $H=-1$. We see oscillatory decay is typical for $|H|<2$. Figure 7 shows the maximum rise $(-y)_{\max }$ for $-2<H<2$ where oscillatory decay forces the sheet to rise above the original level $y=0$. The linear theory under estimates the maximum rise. Figure 8 shows the lateral shortening at infinity. In this case the linear theory is quite inadequate since it predicts zero shortening.

\section{REFERENCES}

[1] M. Hetenyi, Beams on elastic foundations, Univ. of Michigan Press, Ann Arbor, Mich., 1946

[2] S. Timoshenko, Strength of materials, Part II, 3rd ed., Van Nostrand, New York, 1956 